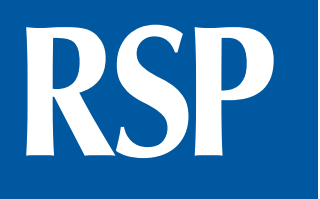

http://www.rsp.fsp.usp.br/
Revista de Saúde Pública

\title{
Avaliabilidade dos Núcleos de Apoio à Saúde da Família com foco na integração às equipes apoiadas
}

\author{
Thaís Titon de Souza', Maria Cristina Marino Calvo" \\ ' Universidade Federal de Santa Catarina. Centro de Ciências da Saúde. Programa de Pós-Graduação em Saúde \\ Coletiva. Florianópolis, SC, Brasil \\ " Universidade Federal de Santa Catarina. Centro de Ciências da Saúde. Departamento de Saúde Pública. \\ Florianópolis, SC, Brasil
}

\section{RESUMO}

OBJETIVO: Analisar a avaliabilidade dos Núcleos de Apoio à Saúde da Família com foco na integração às equipes apoiadas.

MÉTODOS: Estudo de avaliabilidade em que foram realizadas análise documental e modelização da intervenção com foco no trabalho integrado às equipes apoiadas, o que permitiu descrever dimensões, objetivos e resultados esperados. Foram definidos indicadores de resultados e suas respectivas medidas e fontes de informação. Foram aplicadas técnicas de consenso com informantes-chave para validação dos modelos e da matriz de indicadores.

RESULTADOS: O estudo de avaliabilidade mostrou-se apropriado, possibilitando melhor definição e conhecimento acerca da intervenção.

CONCLUSÕES: Há coerência entre os objetivos dos Núcleos de Apoio à Saúde da Família e sua estruturação, ainda que existam dificuldades para operacionalizá-los. É recomendado proceder a uma avaliação formativa dos Núcleos de Apoio à Saúde da Família com foco no trabalho integrado às equipes apoiadas buscando fortalecê-los para o alcance dos resultados esperados.

DESCRITORES: Avaliação em Saúde. Equipe de Assistência ao Paciente. Avaliação de Recursos Humanos em Saúde. Atenção Primária à Saúde, recursos humanos. Sistema Único de Saúde.

Correspondência:

Thaís Titon de Souza

Rodovia Amaro Antônio Vieira,

2008 Bl. 14 Apto. 203 Itacorubi

88034-102 Florianópolis, SC, Brasil

E-mail: thayyts@yahoo.com.br

Recebido: 20 mar 2017

Aprovado: 13 jun 2017

Como citar: Souza TT, Calvo MCM. Avaliabilidade dos Núcleos de

Apoio à Saúde da Família com foco na integração às equipes apoiadas.

Rev Saude Publica. 2018;52:41.

Copyright: Este é um artigo de acesso aberto distribuído sob os termos da Licença de Atribuição Creative Commons, que permite uso irrestrito, distribuição e reprodução em qualquer meio, desde que o autor e a fonte originais sejam creditados. 


\section{INTRODUÇÃO}

Os Núcleos de Apoio à Saúde da Família (NASF) foram criados em 2008 com o objetivo de ampliar a abrangência e o escopo da Atenção Básica (AB), bem como sua resolubilidade ${ }^{1}$. Sua composição deve ser definida a partir das necessidades de saúde do território e das equipes de Saúde da Família e Atenção Básica para Populações Específicas (equipes de $\mathrm{SF} / \mathrm{AB}$ ) apoiadas, com as quais compartilham a responsabilidade pela produção do cuidado.

O apoio matricial orienta o trabalho integrado entre essas equipes. Ações técnico-pedagógicas e assistenciais que melhor respondam às necessidades dos usuários ou do território são definidas com base em pactuações entre os profissionais². A atuação diverge do modelo ambulatorial e da lógica de encaminhamentos indiscriminados, orientando-se pela clínica ampliada e pela corresponsabilização $0^{3,4}$.

Para alcançar a integração e o compartilhamento esperados e, consequentemente, o maior grau de qualidade e resolubilidade possível, são identificados desafios como a insuficiência de mecanismos para monitoramento e avaliação dos resultados alcançados ${ }^{4-6}$. Poucos estudos relatam os resultados das ações do NASF sobre equipes vinculadas e população assistida e estão, em geral, relacionados à sua implantação ou à atuação das diferentes categorias profissionais na $\mathrm{AB}^{7-10}$. A insuficiência de dados oficiais em relação ao trabalho do NASF dificulta a avaliação dos resultados produzidos. Sua inclusão no e-SUS Atenção Básica e no Programa Nacional de Melhoria do Acesso e da Qualidade da Atenção Básica (PMAQ) pode contribuir para superar essa lacuna.

São necessários, portanto, mecanismos de avaliação e monitoramento dos NASF que considerem os resultados alcançados, especialmente a partir do trabalho integrado às equipes vinculadas. Este estudo teve por objetivo analisar a avaliabilidade dos Núcleos de Apoio à Saúde da Família com foco na integração às equipes apoiadas.

\section{MÉTODOS}

Trata-se de um estudo de avaliabilidade (EA), compreendido como um conjunto de procedimentos que precedem a etapa de avaliação propriamente dita e apontam para sua utilidade e oportunidade, tornando-a mais consistente e com maior credibilidade ${ }^{11}$. Dentre os produtos esperados, está a completa descrição do programa ou intervenção em análise e a definição das questões principais a serem consideradas na avaliação ${ }^{12}$.

Utilizou-se abordagem qualitativa. A Figura 1 apresenta o fluxograma de operacionalização. Procedeu-se à análise documental para elaboração dos modelos teórico e lógico do NASF com foco no trabalho integrado às equipes apoiadas. Com base em revisão de documentos do Ministério da Saúde e de literatura especializada das bases Lilacs (Literatura Latino-Americana e do Caribe em Ciências) e SciELO (Scientific Electronic Library Online), foram selecionados nove documentos e 18 artigos científicos. Foram critérios de inclusão: existência de um

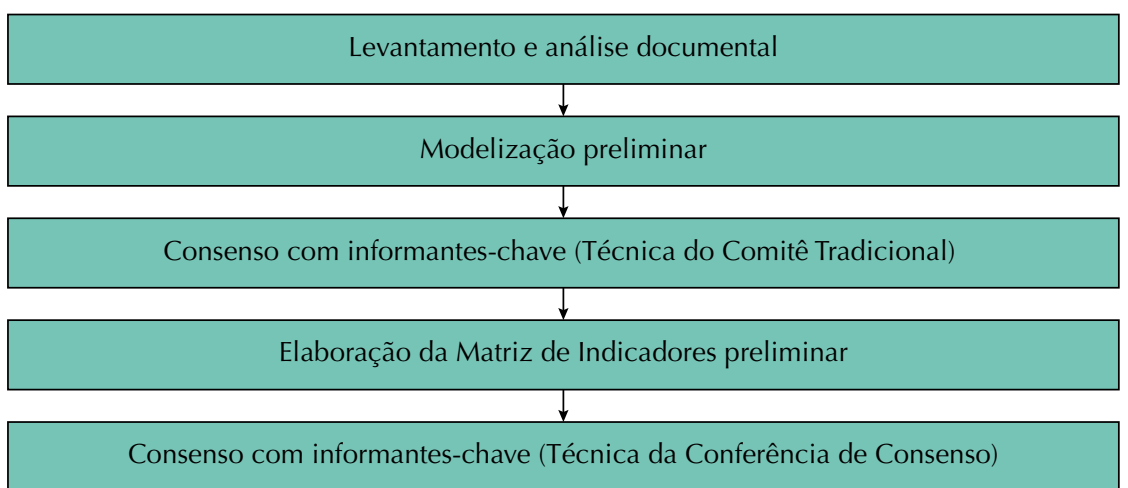

Figura 1. Etapas de delineamento do estudo de avaliabilidade dos Núcleos de Apoio à Saúde da Família. 
ou mais termos de busca (NASF, Núcleo de Apoio à Saúde da Família, apoio matricial ou matriciamento) no título (termos equivalentes não estão disponíveis nos Descritores em Ciências da Saúde [DeCS]); disponibilidade online do texto completo; idioma português; e período de publicação entre 2010 e 2015 para artigos e entre 2004 e 2015 para documentos ministeriais. Não foram considerados materiais sobre aspectos exclusivos de categorias profissionais que compõem o NASF ou experiências de apoio matricial desenvolvidas por outras equipes de saúde e artigos que se repetiram entre as bases.

Após a coleta de informações e a definição clara do objeto em estudo, realizou-se a modelização inicial da intervenção. Para consenso dos modelos, foi utilizada a técnica do Comitê Tradicional, com discussão aberta sobre determinado tema entre especialistas ${ }^{13}$. Foram selecionados 12 informantes-chave, representados por quatro profissionais do NASF, dois profissionais de equipes de $\mathrm{SF} / \mathrm{AB}$, dois gestores em saúde e quatro especialistas em avaliação em saúde, que propuseram mudanças relevantes nos modelos apresentados. Profissionais do NASF, por exemplo, reforçaram a vinculação do NASF à AB e indicaram a necessidade de deixar claros os resultados esperados do trabalho integrado às equipes apoiadas.

Foi elaborada a versão preliminar da matriz de indicadores, validada por meio da Conferência de Consenso, técnica que procura conciliar a possibilidade de discussão aberta e a preservação do anonimato dos participantes ${ }^{14}$. Os especialistas indicaram sua completa concordância ou discordância parcial ou total em relação aos elementos da matriz por meio de um questionário eletrônico, sugerindo alterações. Após a consolidação dos dados, foi realizada uma oficina de consenso presencial para debater questões que não apresentaram concordância na etapa anterior ${ }^{13}$. As contribuições dos especialistas, incluindo profissionais do NASF, foram significativas para esclarecer resultados esperados, redefinir indicadores inicialmente direcionados à avaliação de processo, qualificar medidas e perguntas para a verificação dos indicadores e reestruturar a matriz de maneira a atender ao objetivo de avaliação dos resultados alcançados pelo NASF a partir do trabalho integrado às equipes apoiadas. Por fim, foi reenviado um questionário eletrônico contendo a matriz, obtendo-se consenso final. Participaram dessas etapas 12 informantes-chave, com representação similar à fase de consensualização dos modelos.

A pesquisa foi aprovada pelo Comitê de Ética em Pesquisa com Seres Humanos da Universidade Federal de Santa Catarina (Parecer 1.248.870/2015), com respeito aos preceitos éticos. Todos os participantes tiveram acesso e assinaram o termo de consentimento livre e esclarecido.

\section{RESULTADOS}

\section{Modelo Teórico}

O modelo teórico do NASF orienta o modo como idealmente funciona para alcançar seus objetivos $^{15}$ (Figura 2).

Foram identificados diferentes fatores contextuais que atuam diretamente sobre a $A B$ e interferem na relação estabelecida entre o NASF e equipes apoiadas. As necessidades sociossanitárias da população, por exemplo, exigem dos gestores identificação de prioridades e definição de estratégias para lidar com a realidade. Isso influencia a definição da constituição das equipes do NASF e seu modo de operar em cada território ${ }^{2,3,16}$.

A Constituição Federal brasileira e as legislações que regem o SUS, especialmente a Política Nacional de Atenção Básica, somadas aos diferentes modelos de atenção existentes são também determinantes para a relação estabelecida. Atualmente, predomina o modelo biologicista. Esse modelo orienta o trabalho das equipes de SF/AB para o atendimento individual de grande demanda assistencial, tornando escasso o tempo disponível para discussão, pactuação e o fazer em conjunto. Isso pode pressionar os profissionais de apoio para sua reprodução, conduzindo o NASF ao funcionamento inadequado ${ }^{17,18}$. O descompasso ocasiona resistências e conflitos e dificulta o trabalho colaborativo e a corresponsabilização. 


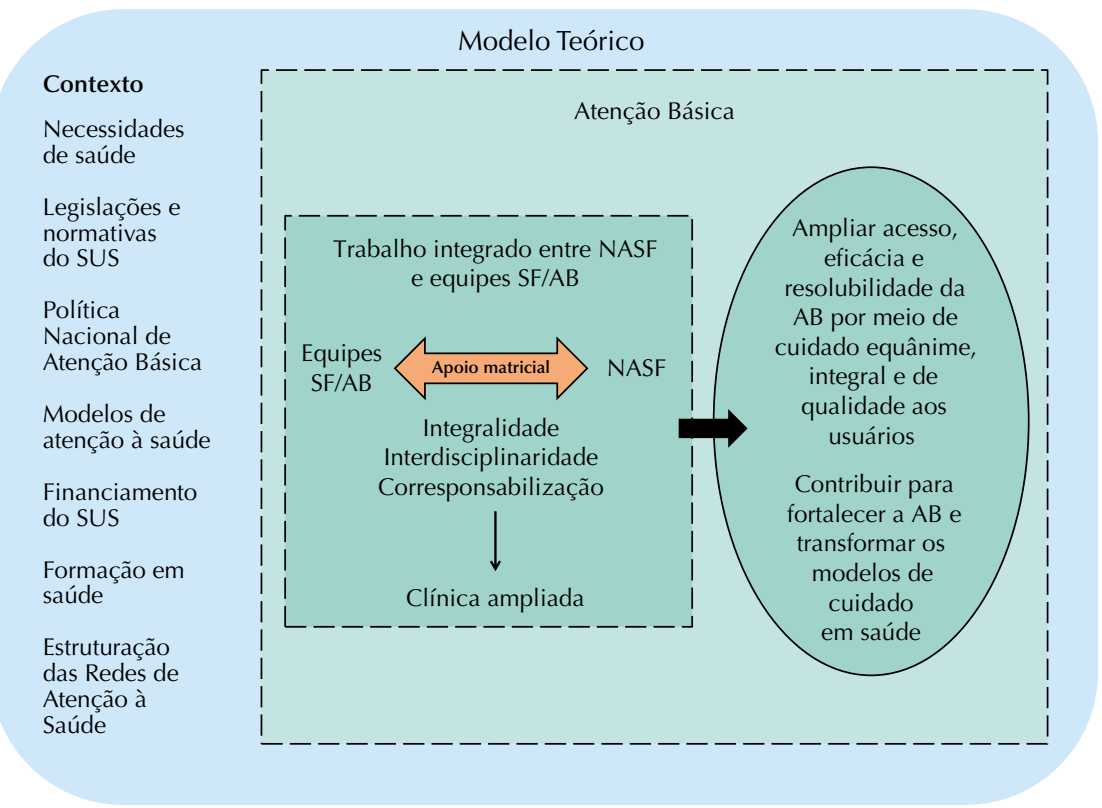

NASF: Núcleo de Apoio à Saúde da Família; Equipes SF/AB: equipes de Saúde da Família e Atenção Básica para Populações Específicas; AB: Atenção Básica; SUS: Sistema Único de Saúde

Figura 2. Representação esquemática do modelo teórico dos Núcleos de Apoio à Saúde da Família.

Somam-se como contexto, a formação profissional comumente deficiente para atuar na lógica do apoio matricial, aspectos relacionados ao financiamento do SUS que interferem diretamente na proposição técnica relacionada à configuração e atuação do NASF (inclusive em relação ao número de equipes apoiadas e população assistida) e a estruturação das Redes de Atenção à Saúde (RAS), especialmente da Média Complexidade ${ }^{4,18,19}$. A incipiência das RAS pode conduzir à desvirtuação da proposta de trabalho integrado ao levar à utilização do NASF como substituto das Redes, direcionando-o apenas à atuação especializada ${ }^{18}$.

Ao contrário dessa lógica, o trabalho integrado entre as equipes ocorre a partir do referencial teórico-metodológico do apoio matricial, considerando-se'2:

- Que o NASF deve atuar de maneira integrada e colaborativa com as equipes de SF/AB nos níveis individual e coletivo de cuidado. Deve ter sob sua responsabilidade a própria equipe apoiada, bem como a assistência direta à população e a atuação sobre o território adscrito ${ }^{23,16}$;

- A integralidade como um preceito para a organização dos serviços de saúde;

- A atuação interdisciplinar, que deve promover o rompimento de relações verticalizadas e minimizar a ocorrência de iatrogenias;

- Que o NASF e equipes apoiadas devam compartilhar a responsabilidade pelos usuários e territórios adscritos, em vez de transferi-la. Isso implica corresponsabilização, norteando ações desenvolvidas em contraste aos modelos baseados na assistência fragmentada e individualizada ${ }^{4} ; \mathrm{e}$,

- Que as dimensões técnico-pedagógica e assistencial do apoio matricial são complementares e podem ser consideradas vertentes práticas da operacionalização dos princípios e das diretrizes do SUS, especialmente da integralidade e da interdisciplinaridade, derivando em ações pautadas na clínica ampliada ${ }^{16}$.

Espera-se a superação da lógica fragmentada e medicalizadora do cuidado em saúde para um cuidado integral, cuja reorientação ocorre de maneira conjunta com a Estratégia Saúde da Família ${ }^{1,20-22}$. Em consequência, o NASF deve apoiar, aperfeiçoar e ampliar a atenção em saúde, fortalecendo os atributos da Atenção Primária à Saúde (APS) junto às equipes apoiadas ${ }^{2-4,7,16,20,23,24}$. 


\section{Modelo Lógico}

Aposta-se que o NASF amplie a abrangência e o escopo da AB, aumente sua capacidade de resposta aos problemas e necessidades de saúde e preste assistência às demandas populacionais não alcançadas pelas equipes apoiadas ${ }^{1,3,4,7,717,20-23}$. Assim, decidiu-se elaborar o modelo lógico para avaliação de seus resultados quanto à ampliação do acesso e da resolubilidade da $\mathrm{AB}$.

Com o intuito de mostrar como a intervenção em estudo deve ser implantada, foram identificadas dimensões, objetivos específicos e resultados esperados a partir do trabalho integrado às equipes de $\mathrm{SF} / \mathrm{AB}$ (Figura 3 ).

A primeira dimensão do modelo apresentou como resultados intermediários a qualificação das equipes apoiadas e a potencialização da clínica ampliada ${ }^{17,21,22,24,25}$. Intervenções técnico-pedagógicas e assistenciais do apoio matricial possibilitam a ampliação de escopo e oferta de ações na $\mathrm{AB}$ por meio da qualificação das equipes para a produção do cuidado e do atendimento às necessidades de saúde anteriormente não atingidas. A incorporação dos profissionais dos NASF, portanto, além de ampliar os recursos humanos, deve possibilitar a oferta de ações com maior proximidade do território e da realidade em que vive a população, por meio do trabalho articulado às equipes de $\mathrm{SF} / \mathrm{AB}^{1-4,7,16,18,20,23}$.

A segunda dimensão, denominada "Resolubilidade", mostrou a intenção de que a implantação do NASF contribua para a ampliação da coordenação e da longitudinalidade do cuidado pelas equipes apoiadas. Para isso, deve favorecer a articulação da $\mathrm{AB}$ com outros pontos das RAS e serviços intersetoriais e qualificar encaminhamentos ${ }^{1,2,25}$.

Ações que aperfeiçoem a utilização da Rede de Atenção secundária e terciária, como a reorganização da demanda e melhoria da relação entre os diferentes pontos de atenção, devem promover racionalização do acesso aos recursos especializados e contribuir para ampliar a capacidade das equipes de SF/AB em coordenar o cuidado dos usuários ${ }^{2,47,77,25}$. Elas colaboram para o fortalecimento do atributo da longitudinalidade, o que tende a produzir diagnósticos e tratamentos mais precisos e reduzir procedimentos de maior densidade tecnológica e encaminhamentos desnecessários para especialistas ${ }^{26}$.

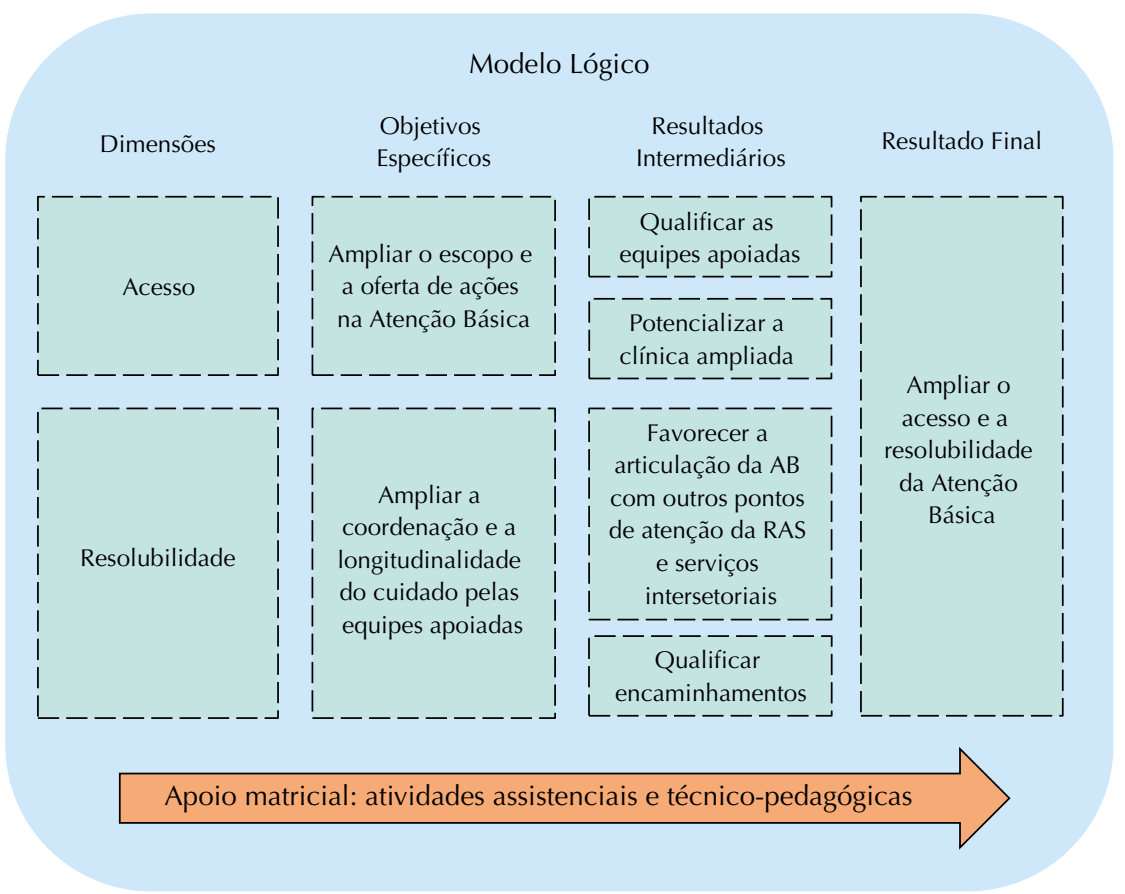

NASF: Núcleo de Apoio à Saúde da Família; AB: Atenção Básica; RAS: Redes de Atenção à Saúde

Figura 3. Representação esquemática do modelo lógico dos resultados alcançados pelos Núcleos de Apoio à Saúde da Família com foco na ampliação do acesso e da resolubilidade na Atenção Básica. 
O apoio do NASF deve favorecer, portanto, a continuidade e a ordenação do cuidado pela $\mathrm{AB}$, fortalecendo-a nas RAS, sem desconsiderar que tais resultados dependem também de outros fatores ${ }^{2,25}$. Sua contribuição para a organização e a redução de encaminhamentos desnecessários a outros serviços constitui-se como uma ação importante frente às limitações da Rede nos diversos pontos de atençãa ${ }^{2,7,18,25}$.

\section{Matriz de Avaliação}

A matriz avaliativa dos resultados dos NASF com foco no trabalho integrado às equipes apoiadas foi construída com base nos modelos teórico e lógico. Apresenta as dimensões "Acesso" e "Resolubilidade", com duas subdimensões e quatro indicadores cada (Quadro).

Na dimensão "Acesso", considerou-se a dupla carga de responsabilidade atribuída ao NASF². Na subdimensão "Atuação das Equipes Apoiadas", buscou-se identificar os resultados da dimensão técnico-pedagógica do apoio matricial sobre a capacidade de produção do cuidado pelas próprias equipes apoiadas por meio de dois indicadores: Prática assistencial: busca identificar o aumento da capacidade das equipes apoiadas para a efetivação do cuidado a partir da análise e atuação em termos clínicos; e, Prática sanitária: objetiva identificar o aumento da capacidade das equipes de SF/AB para a efetivação do cuidado por meio de análise e atuação em termos sanitários e de intervenção coletiva ${ }^{3,720,22,25}$.

Para a composição da subdimensão "Interdisciplinaridade e Integralidade", foram selecionados os indicadores: Integração e compartilhamento entre equipes (verifica os resultados alcançados sobre a capacidade de trabalho interdisciplinar, integrado e colaborativo entre as equipes apoiadas e o NASF para a produção do cuidado); e, Ações ofertadas pelo NASF (avalia resultados alcançados sobre a ampliação do escopo e do acesso às ações assistenciais específicas do NASF, a fim de responder às necessidades e demandas de saúde anteriormente não atendidas pelas equipes de $\mathrm{SF} / \mathrm{AB})$.

Na dimensão "Resolubilidade", considerou-se que a atuação do NASF deve fortalecer a capacidade de coordenação e a oferta de cuidado longitudinal pelas equipes apoiadas, bem como de cooperação e trabalho horizontal com outros pontos de atenção. Por meio de uma lógica que privilegia o trabalho colaborativo, deve potencializar a $\mathrm{AB}$ como ordenadora $\mathrm{e}$ porta de entrada preferencial das RAS, aumentando, inclusive, sua resolubilidade.

Na subdimensão “Coordenação do cuidado”, esperou-se que a responsabilização compartilhada provocasse revisão da prática do encaminhamento a partir da referência e da contrarreferência, ampliando-a para o compartilhamento de responsabilidades ${ }^{3}$. Os indicadores selecionados para essa subdimensão (Gestão terapêutica na Atenção Básica e Articulação da Atenção Básica a outros pontos das RAS e serviços intersetoriais) objetivaram identificar resultados alcançados em relação à capacidade de gestão compartilhada do cuidado entre o NASF e equipes apoiadas e com outros pontos de atenção, além da capacidade de desenvolvimento de ações integradas nas Redes de Atenção e junto aos serviços intersetoriais.

Na última subdimensão "Longitudinalidade", considerou-se que a longitudinalidade exige uma fonte regular de atenção e seu uso ao longo do tempo, independente do tipo de problema ${ }^{26}$. O indicador "Continuidade da relação clínica na AB" teve como objetivo identificar os resultados do NASF sobre a capacidade da $A B$ para sua realização. Isso porque tem como pressuposto a garantia da integralidade da atenção e é um dos aspectos que pode determinar a capacidade em prover o cuidado longitudinal ${ }^{3}$. No âmbito específico da $A B$, o acolhimento em saúde é uma estratégia para o alcance da longitudinalidade pessoal por estar relacionado à boa comunicação e favorecer a continuidade do cuidado, de modo que o NASF possa contribuir para a sua consolidação ${ }^{26}$. Além disso, pode oferecer apoio em situações inesperadas ou que exijam atuação imediata, considerando a capacidade de cada equipe em lidar com essas situações.

A ampliação do rol de profissionais inseridos na $\mathrm{AB}$ possibilitaria também o aumento da capacidade de Resolução clínica, o último indicador apresentado. Além da ampliação dos conhecimentos e práticas profissionais, a mobilidade e a visão do NASF quanto a 
Quadro. Matriz avaliativa dos resultados produzidos por Núcleos de Apoio à Saúde da Família com base no trabalho integrado às equipes apoiadas.

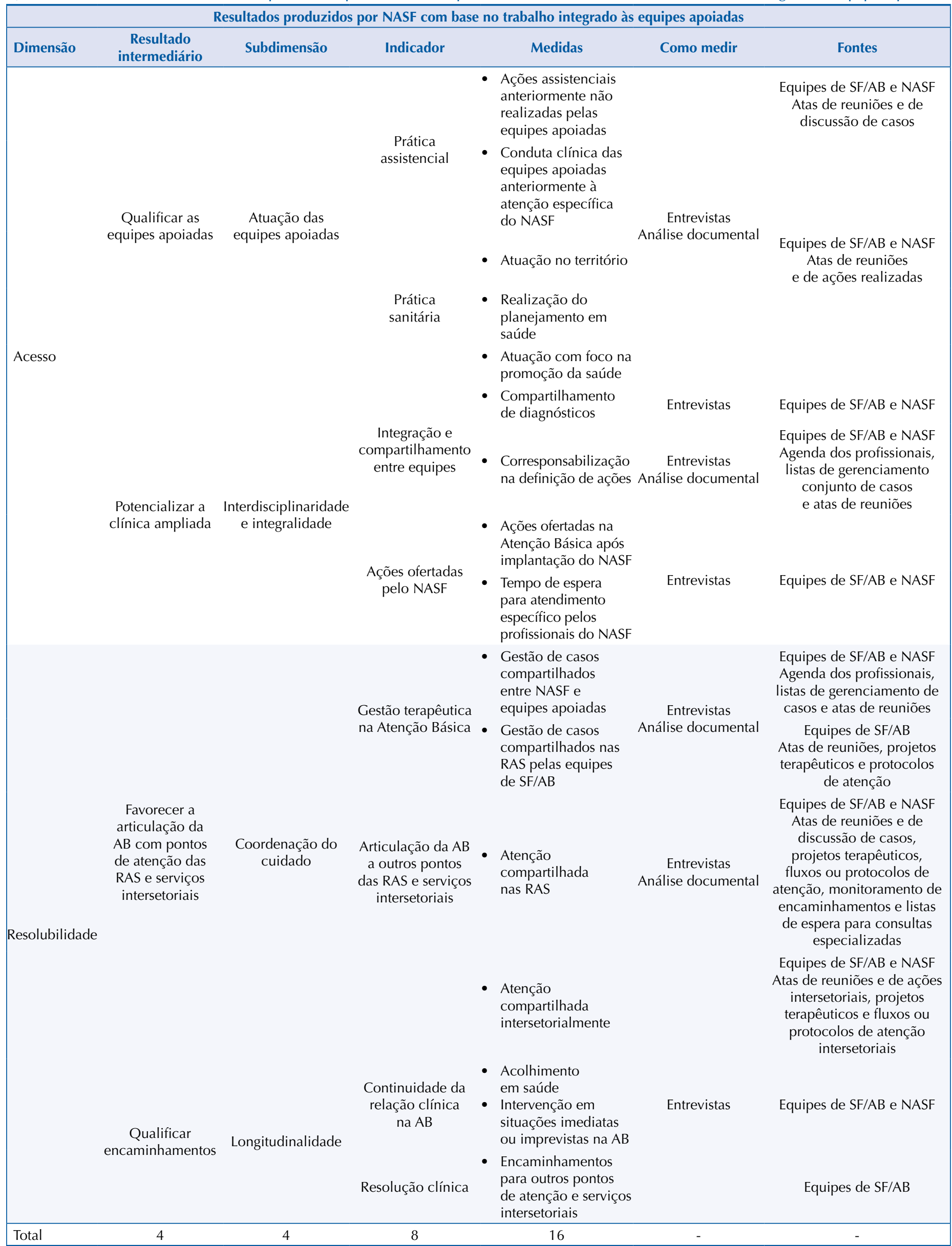

Equipes de SF/AB: equipes de Saúde da Família e Atenção Básica para Populações Específicas; NASF: Núcleos de Apoio à Saúde da Família; AB: Atenção Básica; RAS: Redes de Atenção à Saúde 
diversas equipes favorecem conexões, inclusive intersetoriais, e incrementam projetos terapêuticos, apresentando potencialidade para aperfeiçoar redes e fluxos assistenciais na atuação dessas equipes ${ }^{2}$. Como consequência, deve contribuir para racionalizar o acesso aos serviços especializados de saúde e serviços intersetoriais a partir de ações pactuadas com as equipes de $\mathrm{SF} / \mathrm{AB}^{17}$.

Acesso e resolubilidade foram considerados eixos transversais de observação da matriz avaliativa na medida em que se apresentam como resultados finais esperados e estão envolvidos simultaneamente nas duas dimensões avaliadas. A qualidade transpassou toda a matriz, compreendida como a capacidade de atender aos atributos de valor e mérito, ou seja, de aplicar adequadamente recursos e fazer bem o que se propõe para atender às necessidades das equipes de SF/AB e dos usuários (Scriven, 1991 citado por Scaratti e Calvo, $2012)^{27}$. Ao mesmo tempo em que maior qualidade é atingida quando os resultados finais do trabalho integrado entre o NASF e equipes apoiadas são obtidos, a qualidade também interfere na capacidade dessas equipes em alcançá-los.

\section{DISCUSSÃO}

O pouco tempo de existência do NASF e a insuficiência de estudos avaliativos sobre o tema indicam a importância de pesquisas que contribuam para a compreensão dessa intervenção e para a proposição de questões que direcionem a avaliação formativa dessas equipes ${ }^{11}$.

Na falta de uma teoria explícita e claramente articulada, fez-se necessário desenvolver os modelos teórico e lógico da intervenção ${ }^{11}$. A identificação de seus componentes foi importante para a proposição de indicadores que buscam favorecer o avanço do NASF. A seleção de informantes-chave com conhecimento ou experiência prévia em relação ao tema e em avaliação em saúde foi importante para tornar mais clara a imagem-objetivo pretendida com a implantação dessa equipe, assim como o modo como a intervenção pode ser implantada para alcançá-la.

Tomando como base a análise lógica operacional, percebe-se coerência entre objetivos e estruturação do NASF, ainda que existam dificuldades para operacionalizá-lo. Desafios são encontrados para alcançar integração e compartilhamento esperados a partir do trabalho integrado entre o NASF e equipes apoiadas e, consequentemente, maior grau de qualidade e resolubilidade possível. Alguns deles são: baixa compreensão sobre apoio matricial e sua pouca consolidação prática; dificuldade de definição do objeto de trabalho do NASF; entendimento do NASF como um serviço ambulatorial de especialidades; deficiente formação dos trabalhadores para atuar na lógica preconizada; insuficiência de dispositivos organizacionais para apoiar o trabalho compartilhado; existência de demanda reprimida para algumas categorias profissionais; e insuficiência de mecanismos para monitoramento e avaliação dos resultados alcançados ${ }^{4-6}$.

A insuficiência de mecanismos eficazes de registro e monitoramento que contemplem as ações do NASF torna visível a necessidade de desenvolver instrumentos mensuráveis e eficientes para avaliação e gestão $0^{28}$. Para melhor entendimento sobre o processo de trabalho do NASF e definição de estratégias que possibilitem o alcance dos resultados desejados, faz-se necessário o desenvolvimento de indicadores apropriados. Sua insuficiência ocasiona dificuldades na orientação da atuação em direção à integralidade e à interdisciplinaridade da atenção $0^{28}$.

O estudo de avaliabilidade (EA) deve contribuir para melhorar o desenvolvimento da intervenção analisada. Os modelos teórico e lógico e os indicadores propostos na matriz avaliativa são instrumentos úteis para apoiar a formulação de políticas e a gestão dos NASF no país. Ao guardar estreita relação entre si, explicitam a cadeia de causalidade que leva à obtenção dos resultados esperados e sugere mecanismos para identificar se estão sendo atingidos, indicando sua plausibilidade ${ }^{29}$. O EA desenvolvido, portanto, deve contribuir para aferir se as ações realizadas estão caminhando para obter mudanças esperadas com a implantação dessas equipes na $\mathrm{AB}^{29}$. 
É necessário desenvolver mecanismos de avaliação e monitoramento do trabalho dos NASF e dos resultados produzidos a partir do trabalho integrado às equipes apoiadas. As equipes de SF/AB têm importante papel na consolidação do apoio matricial. Sua organização e compreensão sobre o novo modelo de relação e atenção são primordiais para o arranjo do NASF enquanto uma equipe de apoio na $\mathrm{AB}$ e não como um serviço de especialistas nesse âmbito de atenção. $\mathrm{O}$ modelo avaliativo apresentado se propõe a avaliar os resultados do NASF a partir do trabalho integrado às equipes vinculadas.

O EA mostrou que o NASF é uma intervenção avaliável, na medida em que foi possível ampliar o conhecimento acerca da intervenção e definir componentes e indicadores apropriados. Entretanto, a intervenção não está visivelmente ilustrada nas diferentes literaturas analisadas, que se complementam, mas muitas vezes trazem novos elementos em relação ao que se espera a partir de sua implantação. Há indicativo de falta de clareza sobre o que se espera alcançar a partir de sua atuação integrada às equipes apoiadas. Isso torna imperativa a definição de indicadores apropriados para avaliação dessas equipes.

Os modelos e a matriz apresentados colaboram para avançar nessa questão. O EA se apresenta como uma alternativa para qualificar a compreensão sobre o NASF e para colocar em evidência a identificação e o debate dos resultados alcançados, bem como de estratégias para o seu alcance.

Outros desenhos de avaliabilidade para a intervenção podem ser desenvolvidos, inclusive com foco em uma ou outra dimensão do apoio matricial. Neste estudo, buscou-se ultrapassar essa dualidade a fim de ampliar as possibilidades de atuação do NASF para alcance da qualidade e da resolubilidade esperadas a partir do trabalho integrado às equipes apoiadas.

Não há previsão de controle do contexto externo ao trabalho integrado entre o NASF e equipes apoiadas na matriz proposta, mas ele deverá ser considerado na etapa de avaliação propriamente dita. Condições de trabalho do NASF e equipes apoiadas, estruturação das RAS, composição e tempo de implantação do NASF, dentre outros fatores de contexto, deverão ser ponderados nos estudos de avaliação dos resultados do NASF a partir da integração às equipes apoiadas em diferentes realidades.

Os modelos e a matriz avaliativa partiram da concepção de que a relação entre essas equipes é orientada pelos princípios e diretrizes do SUS e da APS, especialmente pela interdisciplinaridade e integralidade da atenção, promovendo corresponsabilização e colocando em prática a clínica ampliada. Considerando o recente processo de implantação do NASF e a incipiência de mecanismos de avaliação dos resultados, especialmente a partir do trabalho integrado às equipes de $\mathrm{SF} / \mathrm{AB}$, o EA pode contribuir para a qualificação dessa intervenção no país.

\section{REFERÊNCIAS}

1. Ministério da Saúde (BR). Portaria № 154, de 24 de janeiro de 2008. Cria os Núcleos de Apoio à Saúde da Família - NASF. Brasília (DF); 2008 [citado 13 jan 2017]. Disponível em: http://bvsms. saude.gov.br/bvs/saudelegis/gm/2008/prt0154_24_01_2008.html

2. Ministério da Saúde (BR), Secretaria de Atenção à Saúde, Departamento de Atenção Básica. Núcleo de Apoio à Saúde da Família. Vol. 1: ferramentas para a gestão e para o trabalho cotidiano. Brasília (DF), Ministério da Saúde; 2014 [citado 13 jan 2017]. (Cadernos de Atenção Básica, 39). Disponível em: http://bvsms.saude.gov.br/bvs/publicacoes/nucleo_apoio_saude_ familia_cab39.pdf

3. Ministério da Saúde (BR), Secretaria de Atenção à Saúde, Departamento de Atenção Básica. Política Nacional de Atenção Básica. Brasília (DF); 2012 [citado 13 jan 2017]. (Série E. Legislação em Saúde). Disponível em: http://189.28.128.100/dab/docs/publicacoes/geral/pnab.pdf

4. Nascimento DDG, Oliveira MAC. Reflexões sobre as competências profissionais para o processo de trabalho nos Núcleos de Apoio à Saúde da Família. Mundo Saude. 2010 [citado 13 jan 2017];34(1):92-6. Disponível em: https://www.saocamilo-sp.br/pdf/mundo_saude/74/12_ revisao_reflexoes.pdf 
5. Campos GWS, Domitti AC. Apoio matricial e equipe de referência: uma metodologia para gestão do trabalho interdisciplinar em saúde. Cad Saude Publica. 2007;23(2):399-407. https://doi.org/10.1590/S0102-311X2007000200016

6. Costa MML. O Núcleo de Apoio à Saúde da Família (NASF): contextualização de sua implantação na Atenção Básica à Saúde no Brasil. Brasília (DF): Universidade de Brasília; 2013. Trabalho de conclusão de curso de bacharelado em Serviço Social

7. Oliveira IC, Rocha RM; Cutolo LRA. Algumas palavras sobre o NASF: relatando uma experiência acadêmica. Rev Bras Educ Med. 2012;36(4):574-80. https://doi.org/10.1590/S0100-55022012000600019

8. Santos SFS, Benedetti TRB. Cenário de implantação do Núcleo de Apoio à Saúde da Família e a inserção do profissional de Educação Física. Rev Bras Ativ Fis Saude. 2012;17(3):188-94. https://doi.org/10.12820/RBAFS.V.17N3P188-194

9. Scabar TG, Pelicioni AF, Pelicioni MCF. Atuação do profissional de Educação Física no Sistema Único de Saúde: uma análise a partir da Política Nacional de Promoção da Saúde e das Diretrizes do Núcleo de Apoio à Saúde da Família - NASF. J Health Sci Inst. 2012 [citado 13 jan 2017];30(4):411-8. Disponível em: http://www.ceap.br/material/MAT15112013155223.pdf

10. Souza CCBX, Ayres SP; Marcondes EMM. Metodologia de apoio matricial: interface entre a Terapia Ocupacional e a ferramenta de organização dos serviços de saúde. Cad Ter Ocup UFSCar. 2012;20(3):363-8. https://doi.org/10.4322/cto.2012.036

11. Natal S, Samico I, Oliveira LGD, Assis AMJ. Estudo de avaliabilidade da rede de formação de Recursos Humanos da Secretaria de Vigilância em Saúde do Ministério da Saúde. Cad Saude Coletiva. 2010 [citado 13 jan 2017];18(4):560-71. Disponível em: http://www.cadernos.iesc.ufrj. br/cadernos/images/csc/2010_4/artigos/CSC_v18n4_560-571.pdf

12. Thurston WE, Ramaliu A. Evaluability assessment of a survivors of torture program: lessons learned. Can J Program Eval. 2005 [citado 13 jan 2017];20(2):1-25. Disponível em: https://evaluationcanada.ca/secure/20-2-001.pdf

13. Hartz ZMA, organizadora. Avaliação em saúde: dos modelos conceituais à prática na análise da implantação de programas. Rio de Janeiro: Fiocruz; 1997.

14. Souza LEPF, Silva LMV, Hartz ZMA. Conferência de consenso sobre a imagem-objetivo da descentralização da atenção à saúde no Brasil. In: Hartz ZMA, Vieira da Silva LM, organizadoras. Rio de Janeiro: Fiocruz; 2005. p.65-102.

15. Medina MG, Silva GAP, Aquino R, Hartz, ZMA. Uso de modelos teóricos na avaliação em saúde: aspectos conceituais e operacionais. In: Hartz ZMA, Vieira-da-Silva L, organizadoras. Avaliação em saúde: dos modelos teóricos à prática na avaliação de programas e sistemas de saúde. Rio de Janeiro: Fiocruz; 2005. p.41-63.

16. Ministério da Saúde (BR). Diretrizes do NASF: Núcleo de Apoio à Saúde da Família. Brasília (DF); 2009.

17. Cunha GT, Campos GWS. Apoio matricial e Atenção Primária em Saúde. Saude Soc. 2011;20(4):961-70. https://doi.org/10.1590/S0104-12902011000400013

18. Lancman S, Gonçalves RMA, Cordone NG, Barros JO. Estudo do trabalho e do trabalhar no Núcleo de Apoio à Saúde da Família. Rev Saude Publica. 2013;47(5):867-75. https://doi.org/10.1590/S0034-8910.2013047004770

19. Fragelli TBO, Shimizu HE. Competências profissionais para o trabalho do Núcleo de Apoio à Saúde da Família. Rev APS. 2014 [citado 13 jan 2017];17(3):334-44. Disponível em: https://aps.ufjf.emnuvens.com.br/aps/article/view/2213/824

20. Anjos KF, Meira SS, Ferraz CEO, Vilela ABA, Boery RNSO, Sena ELS. Perspectivas e desafios do Núcleo de Apoio à Saúde da Família quanto às práticas em saúde. Saude Debate. 2013;37(99):672-80. https://doi.org/10.1590/S0103-11042013000400015

21. Campos GWS, Figueiredo MD, Pereira Júnior N, Castro CP. A aplicação da metodologia Paideia no apoio institucional, no apoio matricial e na clínica ampliada. Interface (Botucatu). 2014;18 Supl 1:983-95. https://doi.org/10.1590/1807-57622013.0324

22. Moura RH, Luzio CA. O apoio institucional como uma das faces da função apoio no Núcleo de Apoio à Saúde da Família (NASF): para além das diretrizes. Interface (Botucatu). 2014;18 Supl 1:957-70. https://doi.org/10.1590/1807-57622013.0333

23. Ribeiro MDA, Bezerra EMA, Costa MS, Castelo Branco CE, Araújo Neto JD, Moreira AKF, et al. Avaliação da atuação do Núcleo de Apoio à Saúde da Família. Rev Bras Promoç Saude. 2014;27(2):224-31. https://doi.org/10.5020/2426 
24. Bonaldi AP, Ribeiro MD. Núcleo de Apoio à Saúde da Família: as ações de promoção da saúde no cenário da Estratégia Saúde da Família. Rev APS. 2014 [citado 13 jan 2017];17(2):195-203. Disponível em: anhttps://aps.ufjf.emnuvens.com.br/aps/article/view/2072

25. Silva ATC, Aguiar ME, Winck K, Rodrigues KGW, Sato ME, Grisi SJFE, et al. Núcleos de Apoio à Saúde da Família: desafios e potencialidades na visão dos profissionais da Atenção Primária do Município de São Paulo, Brasil. Cad Saude Publica. 2012;28(11):2076-84. https://doi.org/10.1590/S0102-311X2012001100007

26. Oliveira MAC, Pereira IC. Atributos essenciais da Atenção Primária e a Estratégia Saúde da Família. Rev Bras Enferm. 2013;66 No Espec:158-64. https://doi.org/10.1590/S0034-71672013000700020

27. Scaratti D, Calvo MCM. Indicador sintético para avaliar a qualidade da gestão municipal da atenção basca à saúde. Rev Saude Publica. 2012;46(3):446-55. https://doi.org/10.1590/S0034-89102012005000034

28. Rodriguez MR, Leão MA, Souza, NKT. Monitoramento e supervisão do Núcleo de Apoio à Saúde da Família (NASF) em uma região administrativa do Distrito Federal utilizando-se análise de entrevistas. Rev Bras Med Fam Comum. 2014;9(30):38-44. https://doi.org/10.5712/rbmfc9(30)658

29. Bezerra, LCA, Alves CKA, Reis YAC, Samico I, Felisberto E, Carvalho ALB, et al. Identificação e caracterização dos elementos constituintes de uma intervenção: préavaliação da política ParticipaSUS. Cienc Saude Coletiva. 2012;17(4):883-900. https://doi.org/10.1590/S1413-81232012000400011

Contribuição dos Autores: Concepção e planejamento do estudo: TTS, MCMC. Coleta de dados: TTS. Análise, interpretação, redação do trabalho, elaboração e revisão do manuscrito: TTS, MCMC. TTS e MCMC aprovaram a versão final do manuscrito e assumem a responsabilidade pública pelo seu conteúdo.

Conflito de Interesses: Os autores declaram não haver conflito de interesses. 